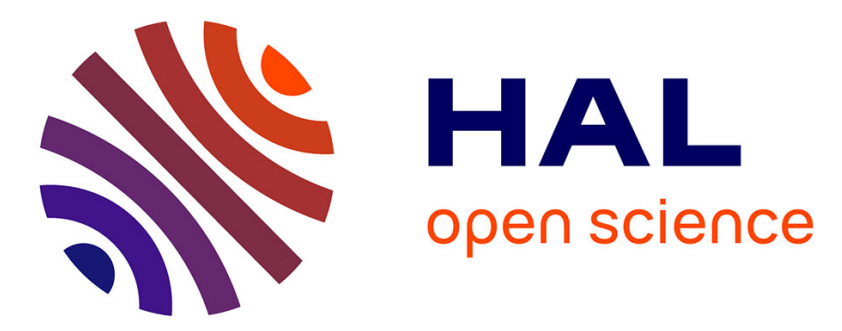

\title{
Dispersion-based pulse shaping for multiplexed two-photon fluorescence microscopy
}

Guillaume Labroille, Rajesh Pillai, Xavier Solinas, Caroline Boudoux, Nicolas

Olivier, Emmanuel Beaurepaire, Manuel Joffre

\section{- To cite this version:}

Guillaume Labroille, Rajesh Pillai, Xavier Solinas, Caroline Boudoux, Nicolas Olivier, et al.. Dispersion-based pulse shaping for multiplexed two-photon fluorescence microscopy. Optics Letters, 2010, 35 (20), pp.3444. 10.1364/OL.35.003444 . hal-00805068

HAL Id: hal-00805068

https://hal-polytechnique.archives-ouvertes.fr/hal-00805068

Submitted on 3 Oct 2013

HAL is a multi-disciplinary open access archive for the deposit and dissemination of scientific research documents, whether they are published or not. The documents may come from teaching and research institutions in France or abroad, or from public or private research centers.
L'archive ouverte pluridisciplinaire HAL, est destinée au dépôt et à la diffusion de documents scientifiques de niveau recherche, publiés ou non, émanant des établissements d'enseignement et de recherche français ou étrangers, des laboratoires publics ou privés. 


\title{
Dispersion-based pulse shaping for multiplexed two-photon fluorescence microscopy
}

\author{
Guillaume Labroille, ${ }^{1}$ Rajesh S. Pillai, ${ }^{1}$ Xavier Solinas, ${ }^{1}$ Caroline Boudoux,${ }^{1,2}$ \\ Nicolas Olivier, ${ }^{1}$ Emmanuel Beaurepaire, ${ }^{1}$ and Manuel Joffre ${ }^{1, *}$ \\ ${ }^{1}$ Laboratoire d'Optique et Biosciences, Ecole Polytechnique, CNRS, and INSERM U696, 91128 Palaiseau, France \\ ${ }^{2}$ Centre d'Optique, Photonique et Laser, Engineering Physics Department, Ecole Polytechnique de Montreal, \\ P.O. Box 6879, Station Centre-Ville, Montreal, Quebec, Canada \\ ${ }^{*}$ Corresponding author: manuel.joffre@polytechnique.edu
}

Received June 29, 2010; revised August 27, 2010; accepted August 30, 2010;

posted September 20, 2010 (Doc. ID 130844); published October 12, 2010

\begin{abstract}
We demonstrate selective two-photon excited fluorescence microscopy with shaped pulses produced with a simple yet efficient scheme based on dispersive optical components. The pulse train from a broadband oscillator is split into two subtrains that are sent through different amounts of glass. Beam recombination results in pulse-shape switching at a rate of $150 \mathrm{MHz}$. Time-resolved photon counting detection then provides two simultaneous images resulting from selective two-photon excitation, as demonstrated in a live embryo. Although less versatile than programmable pulse-shaping devices, this novel arrangement significantly improves the performance of selective microscopy using broadband shaped pulses while simplifying the experimental setup. (c) 2010 Optical Society of America

OCIS codes: $180.4315,320.5540,170.3880$
\end{abstract}

Spectral phase shaping of broadband femtosecond pulses has been shown to be an effective approach for selective two-photon excited fluorescence (2PEF) microscopy [1-5]. Similar to coherent control of two-photon absorption [6,7], selective fluorophore excitation using shaped broadband pulses results from narrowing the laser two-photon spectrum. This is achieved using destructive interference between undesired combinations of pulse spectral components. As compared to the use of a tunable femtosecond laser with a narrower spectral width, one advantage of broadband pulse shaping lies in the ability for fast switching between different pulse shapes, in turn allowing multiplexed addressing of several chromophores. This point is particularly attractive for imaging biological systems [3-5].

In this Letter, we show that the phase-shaping needed for selective $2 \mathrm{PEF}$ microscopy does not necessarily require the use of a programmable pulse shaper as in previous studies but can be achieved using dispersive glass components placed before the microscope. Apart from the advantages of simplicity and efficiency, this simple shaping scheme turns out to be particularly well suited for use in combination with an efficient multiplexing scheme previously demonstrated in the context of multifocal multiphoton microscopy [ $8-10]$ and of coherent antiStokes Raman scattering (CAR $\overline{\text { S }}$ microscopy $[11,12]$. We thus report in vivo dual-excitation $2 \mathrm{PEF}$ imaging in developing embryos with a $5 \mu$ s pixel acquisition time and a switching rate of $150 \mathrm{MHz}$ between the two pulse shapes.

Two-photon excitation efficiency of a chromophore is governed by the overlap between the absorption spectrum of the chromophore and the two-photon spectrum of the pulse (see, e.g., [4,7]). The two-photon spectrum describes the frequencies that the pulse can produce by two-photon excitation and is given by the spectrum of the laser field squared in time domain [4,7]. A strategy for achieving selective two-photon excitation with a broadband pulse is to use a spectral phase profile $\varphi(\omega)$ that is antisymmetric with respect to a particular frequency $\omega_{1}$. The associated group delay $\tau_{1}(\omega)=$ $d \varphi_{1} / d \omega$ is then symmetric with respect to $\omega_{1}$. Frequencies $\omega$ and $2 \omega_{1}-\omega$, symmetric around $\omega_{1}$, therefore correspond to the same group delay and can always mix to contribute to frequency $2 \omega_{1}$ in the two-photon spectrum. In contrast, the production of other frequencies in the two-photon spectrum is strongly inhibited due to the nonsimultaneity of the required frequencies. Various spectral phase profiles suitable for selective two-photon excitation have been previously proposed or demonstrated, including sinusoids $[3,6,7,13]$, quasirandom binary phase masks [14], pseudorandom Galois fields [15], or third-order spectral phase [4,7]. We use here this latter configuration, where the spectral phase reads

$$
\varphi_{1}(\omega)=\frac{1}{6} \varphi^{\prime \prime \prime}\left(\omega-\omega_{1}\right)^{3},
$$

and the corresponding group delay is a parabolic function:

$$
\tau_{1}(\omega)=\frac{d \varphi_{1}}{d \omega}=\frac{1}{2} \varphi^{\prime \prime \prime}\left(\omega-\omega_{1}\right)^{2}
$$

The resulting two-photon spectrum is centered at frequency $2 \omega_{1}$, with a spectral width decreasing when $\left|\varphi^{\prime \prime \prime}\right|$ is increased [4,7]. As shown in this Letter, one advantage of such a third-order spectral phase is that it can be produced by using simple dispersive optical elements instead of a more complex programmable pulse shaper. Indeed, it is well known that a prism compressor produces not only a second-order spectral phase but also a significant amount of third-order spectral phase [16]. After compensation between the second-order terms of opposite signs resulting respectively from the prism compressor and the microscope objective, the net spectral phase is mostly a third-order function of frequency. 


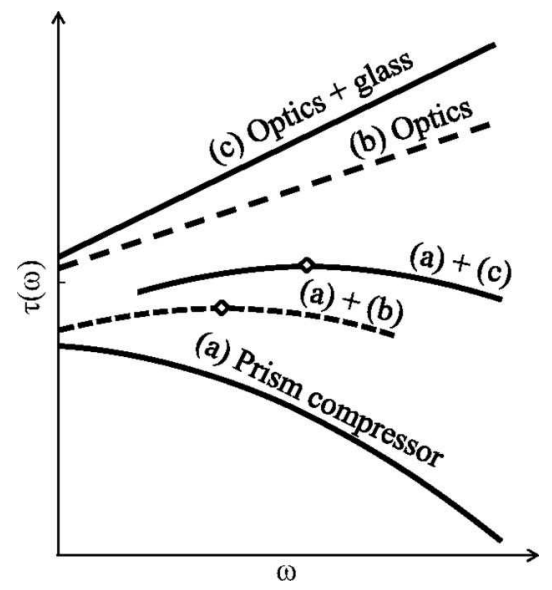

Fig. 1. Schematic plot of the group delay introduced by (a) the prism compressor, (b) the optics of the microscope, and (c) the optics of the microscope plus an additional dispersive glass placed in the beam path, and total group delay without $(a+b)$ and with $(a+c)$ the dispersive glass. The additional dispersion results in a shift of the extremum (diamond).

As shown in Fig. 1, a prism compressor produces a group delay decreasing with frequency but with a strong curvature (a). The remaining microscope optics produce a group delay that exhibits an almost linear increase with frequency (b). Assuming a transform-limited incident laser pulse (constant group delay), the resulting group delay $(a+b)$ is then a parabolic function with a negative curvature, corresponding to Eq. (2). The center frequency of this parabola, $\omega_{1}$, can be easily shifted toward higher frequencies by introducing an additional amount of linear dispersion, e.g., with a piece of glass inserted in the optical path (c). Indeed, let us call $\varphi_{\text {glass }}^{\prime \prime}$ the secondorder phase introduced by the glass. Neglecting higherorder terms that are negligible with respect to those of the prism compressor, the group delay introduced by the glass simply reads $\varphi_{\text {glass }}^{\prime \prime}\left(\omega-\omega_{0}\right)$, where $\omega_{0}$ is the laser center frequency. The total group delay then becomes

$$
\begin{aligned}
& \tau_{2}(\omega)=\tau_{1}(\omega)+\varphi_{\text {glass }}^{\prime \prime}\left(\omega-\omega_{0}\right), \\
= & \frac{1}{2} \varphi^{\prime \prime \prime}\left(\omega-\omega_{1}+\frac{\varphi_{\text {glass }}^{\prime \prime}}{\varphi^{\prime \prime \prime}}\right)^{2}+\text { Cst. }
\end{aligned}
$$

The new parabola $\tau_{2}(\omega)$ is thus centered on frequency $\omega_{2}=\omega_{1}-\varphi_{\text {glass }}^{\prime \prime} / \varphi^{\prime \prime \prime}$, a value greater than $\omega_{1}$ because $\varphi^{\prime \prime \prime}<0$. The final step of rapid switching between

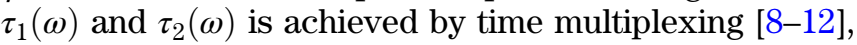
as discussed in the following.

The experimental setup is shown in Fig. 2. The 15 fs pulses produced at a $75 \mathrm{MHz}$ repetition rate by a broadband titanium:sapphire oscillator (Synergy PRO, Femtolasers, Austria) are negatively chirped in a prism-pair compressor ( $\mathrm{N}-\mathrm{SF} 14,40.5 \mathrm{~cm}$ between prisms). The beam is then split in two parts using a half-wave plate and a polarizing beam splitter. The horizontally polarized part of the beam goes straight into the microscope objective $\left(40 \times, 0.8 \mathrm{NA}, \varphi^{\prime \prime} \approx 1970 \mathrm{fs}^{2}, \varphi^{\prime \prime \prime} \approx 1300 \mathrm{fs}^{3}\right)$. In the other path (vertically polarized), the pulses are dispersed through an 11.9-cm-long fused-silica glass piece

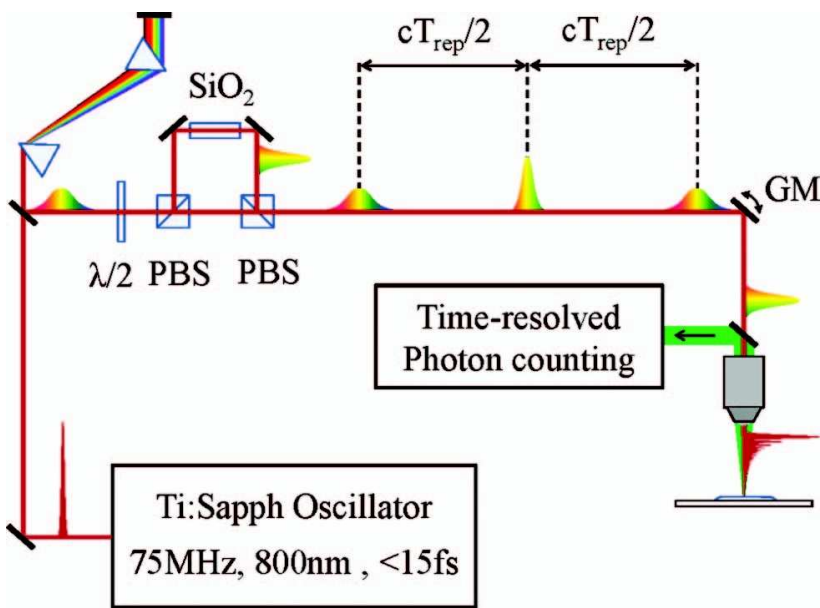

Fig. 2. Experimental setup: HWP, half-wave plate; PBS, polarizing beam splitter; GM, galvanometer-mounted mirrors; $T_{\text {rep }}$, laser period $\left(1 / T_{\text {rep }} \approx 75 \mathrm{MHz}\right) ; c$, speed of light; $c T_{\text {rep }} \approx 4 \mathrm{~m}$.

$\left(\varphi^{\prime \prime} \approx 4300 \mathrm{fs}^{2}, \varphi^{\prime \prime \prime} \approx 3250 \mathrm{fs}^{3}\right)$. This alternate beam is then recombined with the perpendicularly polarized main beam using a second polarizing beam splitter. The alternate path is adjusted to be $2 \mathrm{~m}$ longer than the direct path, so that the corresponding $75 \mathrm{MHz}$ pulse train is exactly interlaced with the $75 \mathrm{MHz}$ pulse train of the main path. This arrangement results in a $150 \mathrm{MHz}$ pulse train with a different pulse shape every other pulse, which corresponds to a $150 \mathrm{MHz}$ switching rate. The $2 \mathrm{PEF}$ is then acquired using a photon-counting scheme synchronized with the $150 \mathrm{MHz}$ pulse train, with two different counters

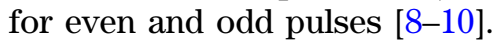

Figure 3(c) shows the two-photon spectra associated with the two pulse shapes, measured with a BBO crystal. Although the two pulses have nearly identical one-photon spectra, their two-photon spectra are strongly focused toward shorter or longer wavelengths, as desired. According to the spacing between the two peaks and to Eq. (4), the corresponding third-order spectral phase of the pulse is roughly $-29000 \mathrm{fs}^{3}$. The small oscillatory feature observed on the high-frequency wing of each spectrum can be shown to originate from the residual fourth-order spectral phase (third-order group delay). Despite this small residual term, Fig. 3 demonstrates that suitable two-photon spectra can be produced at the focus of the microscope objective, despite the lack of an adaptive pulse shaper that would allow a more accurate shaping. Although the throughput of our pulse-shaping scheme was not optimal (55\%) due to some amount of spectral clipping in the prism compressor, we managed to obtain shaped pulses with an average power of about $40 \mathrm{~mW}$ at the focus of the microscope objective. It was then possible to acquire images of biological samples with pixel rates of $120 \mathrm{kHz}$.

Simultaneous dual-excitation imaging capability is demonstrated by observing a developing drosophila embryo, as shown in Fig. 3. The pulses with blueshifted two-photon spectra used for obtaining Fig. 3(a) selectively excite the endogenous fluorophores in the yolk of the embryo, whereas the pulses with redshifted twophoton spectra provide preferential excitation of green fluorescent protein (GFP)-labeled nuclei [Fig. 3(b)]. Figure $3(d)$ is a composite image combining images (a) and $(b)$. The fact that the colors are not pure green and 

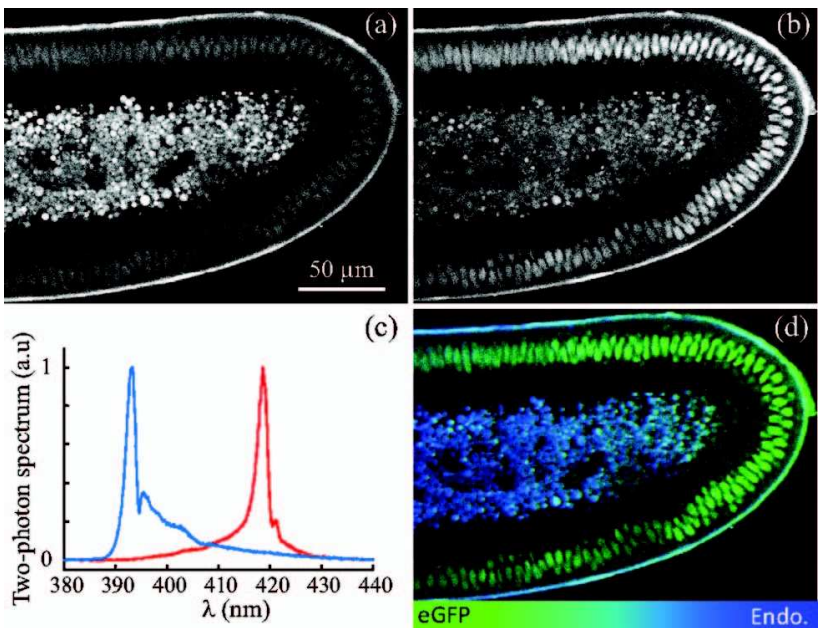

Fig. 3. Imaging with shaped pulses. (a), (b) Drosophila images obtained with blueshifted pulses preferentially exciting (a) blue endogenous fluorescence and (b) redshifted pulses preferentially exciting GFP. (c) Corresponding two-photon spectra measured with a BBO crystal for the blueshifted and redshifted pulses. (d) Combination of (a) and (b) according to the color scale. Refer to movie file (Media 1).

blue indicates that there is some excitation crosstalk between the two channels. This can be attributed to several factors, including the overlap between the $2 \mathrm{PEF}$ excitation spectra of the two fluorophores, the excited state lifetimes of the fluorophores in the range of several nanoseconds, and the response time of the photomultiplier and of the switching electronics. Because the images are truly simultaneous, the contrast can be improved by linear combinations using prior measurement of the relative excitation efficiency of the two chromophores [3,4]. Higher contrast would also be obtained in the case of faster signals (e.g., harmonic generation) or lower laser repetition rates.

To summarize, we have demonstrated a new pulseshaping scheme for in vivo selective two-photon excited fluorescence microscopy based on time multiplexing and dispersive optical elements. Although the purpose of this shaping approach is clearly not to generally compete with more versatile conventional programmable pulse shapers, we have shown that it was compatible with the delivery of broadband pulses at the focus of a microscope objective with a spectral phase suitable for selective 2PEF microscopy. Furthermore, the fact that the second pulse shape was obtained from the first one by a simple additional dispersion enabled time multiplexing, allowing a $150 \mathrm{MHz}$ switching rate between the two shapes. As compared to previous implementations of 2PEF microscopy with shaped broadband pulses, the setup demonstrated here is thus simpler and benefits from a much higher switching rate. Finally, we note that this pulse-shaping scheme could be directly incorporated in multiphoton microscopes employing even broader bandwidth laser sources.

We thank Emmanuel Farge for providing us with the transgenic eGFP labeled Drosophila strain. We thank Delphine Débarre and Pierre Mahou for fruitful comments. This work was supported by Agence Nationale de la Recherche (ANR-06-PCVI-0013), the Conseil Général de l'Essonne (Astre 2006), and Fondation Louis D. de l'Institut de France.

\section{References}

1. I. Pastirk, J. D. Cruz, K. Walowicz, V. Lozovoy, and M. Dantus, Opt. Express 11, 1695 (2003).

2. J. D. Cruz, I. Pastirk, M. Comstock, V. Lozovoy, and M. Dantus, Proc. Natl. Acad. Sci. U.S.A. 101, 16996 (2004).

3. J. P. Ogilvie, D. Debarre, X. Solinas, J.-L. Martin, E. Beaurepaire, and M. Joffre, Opt. Express 14, 759 (2006).

4. R. S. Pillai, C. Boudoux, G. Labroille, N. Olivier, I. Veilleux, E. Farge, M. Joffre, and E. Beaurepaire, Opt. Express 17, 12741 (2009).

5. K. Isobe, A. Suda, M. Tanaka, F. Kannari, H. Kawano, H. Mizuno, A. Miyawaki, and K. Midorikawa, Opt. Express 17, 13737 (2009).

6. D. Meshulach and Y. Silberberg, Nature 396, 239 (1998).

7. V. Lozovoy, I. Pastirk, K. Walowicz, and M. Dantus, J. Chem. Phys. 118, 3187 (2003).

8. W. Amir, R. Carriles, E. Hoover, T. Planchon, C. Durfee, and J. Squier, Opt. Lett. 32, 1731 (2007).

9. R. Carriles, K. Sheetz, E. Hoover, J. Squier, and V. Barzda, Opt. Express 16, 10364 (2008).

10. E. Chandler, E. Hoover, J. Field, K. Sheetz, W. Amir, R. Carriles, S. Ding, and J. Squier, Appl. Opt. 48, 2067 (2009).

11. I. Rocha-Mendoza, W. Langbein, P. Watson, and P. Borri, Opt. Lett. 34, 2258 (2009).

12. W. Langbein, I. Rocha-Mendoza, and P. Borri, Appl. Phys. Lett. 95, 081109 (2009)

13. J. D. Cruz, I. Pastirk, V. Lozovoy, K. Walowicz, and M. Dantus, J. Phys. Chem. A 108, 53 (2004).

14. M. Comstock, V. Lozovoy, I. Pastirk, and M. Dantus, Opt. Express 12, 1061 (2004).

15. V. Lozovoy, B. Xu, J. Shane, and M. Dantus, Phys. Rev. A 74, 041805 (2006).

16. R. Fork, O. Martinez, and J. Gordon, Opt. Lett. 9, 150 (1984). 